\title{
Editorial
}

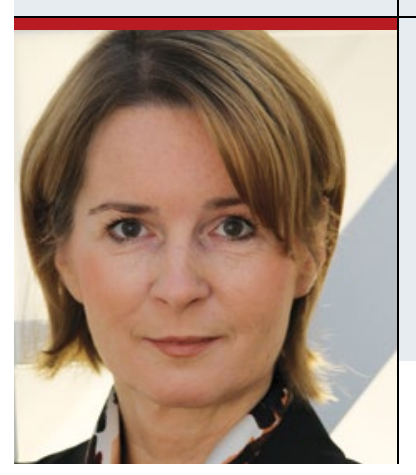

Katja Kupfer Geißler

Chefredaktion

katja.kupfer@springer.com

facebook.com/heilberufe

\section{Auf dem Vormarsch: Krätze}

$\mathrm{P}$ arasiten kenne ich nur vom Hörensagen: Wurmkuren von meiner Mutter aus der Nachkriegszeit, Läuse hatten die Kinder von Freundinnen, erworben in Kindergarten und Schule. Und Krätze - Wer hat das denn heute noch? Anscheinend eine Menge Menschen. Die WHO schätzt den Anteil auf 300 Millionen, die an Krätze (Scabies) erkrankt sind. Die Krätzemilben werden durch Hautkontakt übertragen, bohren bei ihren Opfern winzige Gänge in die Haut, wo sie dann ihre Eier und Ausscheidungen deponieren und unser Immunsystem mit einer Entzündung reagieren lassen. Die Infektionskrankheit verbreitet sich vor allem dort, wo Menschen eng zusammen leben, beispielsweise in Altenheimen. Ein Handtuch, eine Umarmung reicht schon zur Übertragung. Ein Krätzeausbruch wächst sich somit zu einer echten Herausforderung aus, gilt doch hier das Motto „alle für einen“. Denn die Behandlung müssen alle - Bewohner, Pflegende und die Angehörigen - über sich ergehen lassen. Unser Autor Dr. Wulf-Thorsten Gerdts beschreibt im PflegeKolleg Krätze, Läuse \& Co. (Seite 9) anschaulich, wie man die Folgen eines Krätzeausbruchs in den Griff bekommt.

\section{Viel Freude bei der Fortbildung mit HEILBERUFE}

Ihre

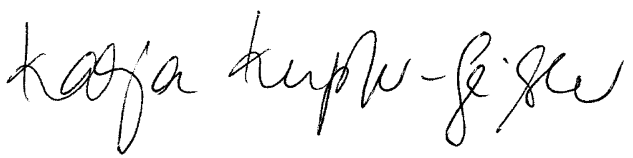

\section{SPRINGER GOES PFLEGE - UND DIE BRANCHE GRATULIERT}

\section{Das durchweg positive Feedback auf die Etablierung unserer Marke Springer Pflege hat uns sehr gefreut. Einige Reaktionen:}

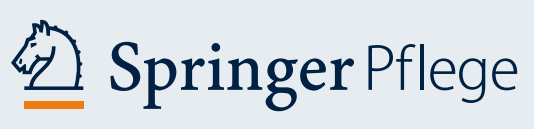

Ich gratuliere herzlich zu dieser guten Entscheidung, die ich persönlich begrüße und sehr gerne unterstütze.

H. François-Kettner, Berlin

Ich gratuliere ganz herzlich zur erfolgreichen Gründung der Marke Springer Pflege und wünsche Ihnen viel Erfolg auf diesem Weg. Gerne möchte ich ganz persönlich auch meinen Teil dazu beitragen, Springer Pflege zu einem Erfolg zu machen.

B. Zieger, Hannover

Springer Pflege - das klingt gut! Ich wünsche Ihnen allen einen erfolgreichen, pflegeorientierten, sich entwickelnden und inspirierenden Weg. Es freut mich, dass die Pflege bei Ihnen einen eigenen "Raum" bekommt. Jetzt müssen wir weiter daran arbeiten, dass die Pflege im politischen und gesellschaftlichen Feld diesen auch erhält.

C. Vogler, Berlin

„Springer Pflege - Das finde ich sehr gut! Sehr gern würde ich Aktivitäten, wenn gewünscht, mit unterstützen."

K. Fuchs, Dresden "Kondition eines Langstreckenläufers und am Ende wird Springer in der Pflege genauso kompetent sein, wie in der Medizin - gratuliere!"

G. Ralle, München 